\title{
Post-ISCO Ringdown Amplitudes in Extreme Mass Ratio Inspiral
}

\author{
Shahar Hadar and Barak Kol \\ Racah Institute of Physics, Hebrew University \\ Jerusalem 91904, Israel \\ E-mail: shaharhadar@phys.huji.ac.il, barak_kol@phys.huji.ac.il
}

\begin{abstract}
An extreme mass ratio inspiral consists of two parts: adiabatic inspiral and plunge. The plunge trajectory from the innermost stable circular orbit (ISCO) is special (somewhat independent of initial conditions). We write an expression for its solution in closed-form and for the emitted waveform. In particular we extract an expression for the associated black-hole ringdown amplitudes, and evaluate them numerically.
\end{abstract}




\section{Contents}

1. Introduction and summary 1

2. Plunge trajectory 4

$\begin{array}{lll}3 . & \text { Source for gravitational waves } & 6\end{array}$

3.1 A general source in the Schwarzschild background 6

$\begin{array}{ll}3.2 & \text { Plunging mass as a source } \\ \end{array}$

4. Solving the wave equation 11

$\begin{array}{ll}4.1 \text { Green's function } & 12\end{array}$

$\begin{array}{lll}4.2 & \text { Ringdown amplitudes } & 13\end{array}$

5. Numerical Evaluation $\quad \mathbf{1 5}$

$\begin{array}{lll}5.1 \text { Method } & 15\end{array}$

$\begin{array}{lll}5.2 & \text { Results } & 17\end{array}$

\section{Introduction and summary}

Gravitational wave observatories (see for example the reviews [1] and references therein) demand knowledge of the waveform emitted by an inspiralling binary system of compact objects. Analytic control is possible in two limits. The first is the Post-Newtonian (PN) approximation, which holds whenever the velocities are small compared with the speed of light. The second limit, the one relevant to this paper, is that of an extreme mass ratio (EMR), for example, a compact projectile such as a stellar size black hole (BH) falling into a super-massive black hole. Numerical solutions complement the analytic methods and have become possible in recent years - see the review [2] and references therein. However, while numerical simulations work very well for comparable masses, they encounter problems in the EMR case due to the existence of two disparate scales which proves problematic for the discretized grid. So it happens that just where the numerical method loses precision, the analytic EMR approximation becomes more effective. Moreover, analytic methods improve insight into the problem, especially into its dependence on parameters.

The evolution of an Extreme Mass Ratio Ispiral (EMRI) is customarily divided into two stages: an adiabatic inspiral where the system moves on quasi-bound orbits and slowly loses energy to gravitational waves, and a plunge phase where the system is set on a course of collision even when the self-force is neglected. In this limit the plunge phase simplifies to consist of geodesic motion of the projectile till it reaches the horizon of the larger $\mathrm{BH}$. The ISCO (innermost stable circular orbit) is the border between the two stages, after which 
the compact object freely falls into the horizon, followed by merger and ringdown (through quasi-normal modes) into a new stationary state. The smooth transition between adiabatic inspiral and plunge was beautifully understood in [3]. While the adiabatic inspiral is well within the applicability domain of the heavily studied PN approximation, known today up to order 3.5PN (as summarized in the review [4]) considerably less is known analytically about the plunge phase which is the subject of this paper.

We would like to have a mathematical description of the waveform throughout its evolution, beginning with the adiabatic inspiral and lasting until the waveform vanishes after the black hole rings down and reaches steady state.

The current state-of-the-art for such an all-time analytic expression goes under the name "Effective One Body formalism" (EOB) [5, 6], see also the recent review [7]. ${ }^{1}$ The EOB approach combines quite a number of ideas and ingredients, but here it will suffice to concentrate on its handling of the ringdown phase, which is quite independent of the other ingredients, and is based on the following assumption: the modeled waveforms during the adiabatic and the ringdown phases may be matched together without the need for an independent modeling of an intermediate phase. This assumption was later vindicated by full numerical simulations. More specifically, the adiabatic phase is carefully modeled and a general expression for the ringdown phase is matched directly onto it. In [8] the full waveform was computed in the extreme mass ratio limit by numerically evolving the trajectory within the EOB approach and numerically determining the radiation. In [9] the model evolved to its present form where the waveform emitted during ringdown is modeled by the most general ringdown signal - a linear superposition of its quasi-normal modes (eq. (18) there)

$$
\Psi_{22}^{\text {ringdown }}(t)=\sum_{n} R_{n}^{+} e^{-\sigma_{n}^{+} t}+\sum_{n} R_{n}^{-} e^{-\sigma_{n}^{-} t}
$$

where $\sigma_{n}^{ \pm} \equiv \alpha_{n} \pm i \omega_{n}$ are positive/negative quasi-normal-mode (QNM) complex frequencies, $R_{n}^{ \pm}$are the corresponding ringdown amplitudes, and $n$ labels the QNM overtone number. This matching is implemented by matching the EOB model for the adiabatic phase with a general ringdown waveform $(1.1)^{2}$ around some judiciously chosen matching time ${ }^{3}$ matching at as many time points as the number of amplitudes to be determined.

In this paper we shall compute the ringdown amplitudes directly from the theory for a certain special plunge trajectory. ${ }^{4}$ For simplicity we consider the trajectory to be moving in the background of a non-rotating (Schwarzschild) $\mathrm{BH}$, and we moreover consider the

\footnotetext{
${ }^{1}$ The method's main idea is an attempt to carry over to General Relativity (GR) the successful classical reduction of the two body problem to a reduced one body problem, where here in GR the reduced point particle moves in some "corrected" metric background. This background does not solve Einstein's equations and as such seems to offer enough adjustable parameters to fit for the sought waveforms. Its proven success makes it undisputable as an ad-hoc method. Yet it is not obvious that it is theoretically justified or natural, in the sense that a natural approximation would not contain unnecessary adjustable parameters nor would it lack parameters for higher order approximations.

${ }^{2}$ With $R_{n}^{-}=0$.

${ }^{3}$ The crossing of the "light ring" at $r=1.5 r_{s}$.

${ }^{4} \mathrm{~A}$ similar approach was taken in [10]. However they differ significantly from the current study both in focus and in results. In particular, they do not calculate the ringdown amplitudes.
} 
plunge trajectory to be the one which starts at the innermost stable circular orbit (ISCO) at $r_{\mathrm{ISCO}}=3 r_{s}$ (in Schwarzschild coordinates, where $r_{s}$ is the Schwarzschild radius). This trajectory is special since the eccentricity of an orbit is known to decrease during the Keplerian regime $r>r_{s}$ of the inspiral [11], thus being a "Keplerian attractor" and hence only weakly dependent on initial conditions. In the case that the inspiral has been going on for enough time for the eccentricity to be essentially radiated away by the time ISCO is reached, then the plunge trajectory will indeed start from roughly ISCO. However, there are relatively recent indications that in some probable astrophysical scenarios other initial conditions are expected, namely initial conditions such that the last stable orbit is not circular, see for example $[12,13,14]$. Yet, in this paper we confine ourselves to the post-ISCO plunge for its simplicity and for being a Keplerian attractor.

For the post-ISCO plunge trajectory we compute the form of the emitted gravitational waves. In the $t \rightarrow \infty$ limit we may extract the post-ISCO ringdown amplitudes, while the formal limit $t \rightarrow-\infty$ corresponds to radiation from ISCO. We wish to stress that the waveform under study continuously interpolates between two time regions: in early time the compact object is near ISCO and the radiation is characterized by the frequency of that trajectory, in the transition period the trajectory is not periodic and hence there are no sharp frequencies in the waveform, while at late times the decaying radiation is characterized by the (complex) frequencies of the quasi-normal modes which are set by the black hole, and not by the trajectory.

In section 2 we solve analytically for the plunge trajectory, namely the trajectory with energy and angular momentum identical to the ISCO values. The expression for $r=r(\phi)$ is known (at least since [15]) to be especially simple and the time dependence can also be expressed in closed form $(2.10,2.12)$. In section 3 we review the Zerilli/Regge-Wheeler $[16,17]$ theory for radiation in the background of a Schwarzschild $\mathrm{BH}$ for a given source of energy-momentum (using Martel-Poisson [18]). We proceed to substitute in the energymomentum for our post-ISCO plunge trajectory. In section 4 we write a formal solution to the wave equation in terms of Green's functions (4.12). We extract the late-time ringdown form by deforming a frequency integral into the complex domain and transforming it to a sum over residues at the quasi-normal modes. The final expression for each post-ISCO ringdown amplitude (4.18) calls for solving certain ordinary differential equations (the radial wave equations) at the QNM frequencies and performing a certain weighted radial integral over the source. In section 5 we numerically evaluate these integrals and obtain the amplitudes of the leading ringdown modes shown in tables 1,2 and in figure 3. These values were confirmed by full numerical simulations [30].

Our expression (4.12) contains more than the ringdown amplitudes: it describes the full plunge waveform as a function of time. Solving for all $(l, m)$ is equivalent to solving an inhomogeneous $3+1$ wave equation and extracting the asymptotic outgoing signal.

The impact of our work is that hereafter the EMRI post-ISCO ringdown amplitudes should be considered to be determined and known (at least under the assumptions above) and any waveform model must conform with it at $t \rightarrow \infty$ (and even as early as ISCO is crossed assuming the full plunge waveform is used rather than only its late time part).

Generalizations. Several generalizations remain. First, one may consider the back- 
ground to be a rotating $\mathrm{BH}$ (Kerr), in which case there are two kinds of post-ISCO plunge trajectories: co-rotating and counter-rotating, and the Zerilli/Regge-Wheeler equations must be replaced by the Teukolsky equation. Second, one may consider orbits where eccentricity (and being off the equator plane in the Kerr background) was not washed away yet. Finally higher order effects may be incorporated such as accounting for the projectile's spin.

\section{Plunge trajectory}

Consider an inspiralling binary system in the Extreme Mass Ratio Inspiral (EMRI) limit, namely $\tilde{m} \ll M$ where $\tilde{m}$ is the mass of the compact object falling into a $\mathrm{BH}$ with mass $\mathrm{M}$, or Schwarzschild radius $r_{s}=2 G M .^{5}$ In order to calculate the gravitational radiation emitted from a system we need to find the dynamics of the compact source. For reasons to be discussed below we consider the (geodesic) trajectory which spirals out of the innermost stable circular orbit (ISCO) at $r_{\mathrm{ISCO}}=3 r_{s}$ and plunges into the black hole. In this section we shall solve for this trajectory.

We concentrate on the case when the inspiral has been going on long enough for the radiation reaction force to have circularized the trajectory by the time the compact object reaches the innermost stable circular orbit (ISCO) at $r_{\mathrm{ISCO}}=3 r_{s}$. This assumption deserves a discussion. Peters [11] found that in the Keplerian regime $r \gg r_{\text {ISCO }}$ energy and angular momentum are lost such that eccentricity decreases with the following rate

$$
p=c_{0} e^{\frac{12}{19}}\left(1+\frac{121}{304} e^{2}\right)^{\frac{870}{2299}}
$$

where $p$ is the Keplerian semi-latus rectum, and $e$ is the eccentricity, such that the trajectory is given by $r(\theta)=p /(1+e \cos (\theta))$ and the semi-major axis $a$ is given by $a=p /\left(1-e^{2}\right)$. Assuming small $e$ and extrapolating (2.1) down to ISCO we can estimate that

$$
e_{f} \simeq e_{i}\left(\frac{r_{\mathrm{ISCO}}}{p_{i}}\right)^{\frac{19}{12}}
$$

where $f, i$ subscripts denote final and initial, respectively. Note that this is only an approximation since the radiation reaction force is different in the strong gravity regime $r \gtrsim r_{\text {ISCO }}$, and it is actually known that $e$ increases somewhat just before ISCO [20]. Altogether, (2.1) and (2.2) quantify the rate of circularization and explain why $e$ would be small at ISCO given $p_{i} \gg r_{\mathrm{ISCO}}$. Hence ISCO is a Keplerian attractor. Yet, as mentioned in the introduction it should be borne in mind that initial conditions such that the last stable orbit is not circular are also to be expected.

Since ISCO is a (marginally) unstable orbit, we choose the plunging orbit with the same conserved parameters as those at ISCO, namely

$$
\begin{aligned}
& \tilde{E}=\tilde{E}_{\mathrm{ISCO}} \equiv \frac{2 \sqrt{2}}{3} \\
& \tilde{L}=\tilde{L}_{\mathrm{ISCO}} \equiv \sqrt{3} r_{s},
\end{aligned}
$$

\footnotetext{
${ }^{5}$ We reserve the letter $m$ to denote the magnetic number - an index for the spherical harmonic functions $Y^{l m}$.
} 
where $\tilde{E}_{\mathrm{ISCO}}, \tilde{L}_{\mathrm{ISCO}}$ are the particle's energy per unit mass and angular momentum per unit mass at ISCO. During the plunge radiation reaction can be neglected as the object is already falling "without its help". ${ }^{6}$

We wish to find explicitly the trajectory of this free-fall. Without loss of generality the trajectory can be considered to lie in the equatorial plane and the equations governing the object's motion are

$$
\begin{aligned}
\tilde{E} & =f(r) \frac{d t}{d \tau} \\
\tilde{L} & =r^{2} \frac{d \phi}{d \tau} \\
\tilde{E}^{2} & =\left(\frac{d r}{d \tau}\right)^{2}+f(r)\left(\tilde{L}^{2} / r^{2}+1\right),
\end{aligned}
$$

where $f(r)=1-\frac{r_{s}}{r}$.

In our special orbit, there is a simple relation between the $r$ and $\phi$ coordinates of the particle (noticed at least as early as [15])

$$
\frac{r_{\mathrm{ISCO}}}{r}=1+\frac{12}{\left(\phi-\phi_{0}\right)^{2}},
$$

where $\phi_{0}$ is an arbitrary constant (sometimes we will choose it to be zero). We will also use conventions such that $\frac{d \phi}{d \tau}>0$.

We proceed to solve for the relation between $t$ and $r$ through integration of equation (2.6), using equation (2.4) to change the derivative with respect to $\tau$ to a derivative with respect to $t$. For a generic orbit we can solve for $t=t(r)$ by quadrature involving an elliptic integral

$$
\tilde{E} \int \frac{d r}{f(r) \sqrt{\tilde{E}^{2}-f(r)\left(\frac{\tilde{L}^{2}}{r^{2}}+1\right)}}=-\int d t .
$$

For our special orbit, the expressions simplify due to the special form of the effective potential (namely that both its first and second derivatives vanish at $r_{\text {ISCO }}$ ). Plugging in our constants of motion, $\tilde{E}_{\mathrm{ISCO}}$ and $\tilde{L}_{\mathrm{ISCO}}$, it can be seen that (2.6) becomes

$$
\left(\frac{d r}{d \tau}\right)^{2}=\frac{1}{3^{2}}\left(\frac{r_{\mathrm{ISCO}}}{r}-1\right)^{3}
$$

This obviously simplifies the integrand, and with a change of variables $\chi:=\frac{1}{2}\left(\frac{r_{\text {ISCO }}}{r}-1\right)$ it is brought to the form of a rational function. Evaluating the integral,

\footnotetext{
${ }^{6}$ The leading corrections for the ISCO values at plunge were computed in [3] eq. (3.26) and they were found to be proportional to $(\tilde{m} / M)^{4 / 5}$.
} 
we find that our special orbit satisfies the following relations

$$
\begin{aligned}
t(r) / r_{s} & =\frac{2 r\left(1-12 \frac{r_{s}}{r}\right)}{r_{s} \sqrt{\chi}}-22 \sqrt{2} \tan ^{-1}(\sqrt{2 \chi})+2 \tanh ^{-1}(\sqrt{\chi})+t_{0} \\
t(\phi) / r_{s} & =3 \sqrt{6}\left(\phi-\phi_{0}\right)\left(1+\frac{4}{\left(\phi-\phi_{0}\right)^{2}+12}\right)-22 \sqrt{2} \tan ^{-1}\left(\frac{2 \sqrt{3}}{\phi_{0}-\phi}\right) \\
& +2 \tanh ^{-1}\left(\frac{\sqrt{6}}{\phi_{0}-\phi}\right)+t_{0}
\end{aligned}
$$

where $t_{0}$ is an arbitrary constant which we shall fix in section 5 . We note that $\chi$ is closely related to the standard change of variables in Kepler's problem $u:=1 / r$ and that $\chi$ varies from $\chi=0$ at ISCO to $\chi=1$ at the horizon.

\section{Source for gravitational waves}

In this section we proceed to describe the equation satisfied by the emitted gravitational waves given a source moving on the geodesic trajectory described in the previous section.

\subsection{A general source in the Schwarzschild background}

The theory of linearized perturbations of Schwarzschild space-time was initially developed in the pioneering works of Regge and Wheeler (1957) [16] and of Zerilli (1970) [17]. Essentially, when expanding the metric perturbations in spherical harmonics, the physics of the

(a)

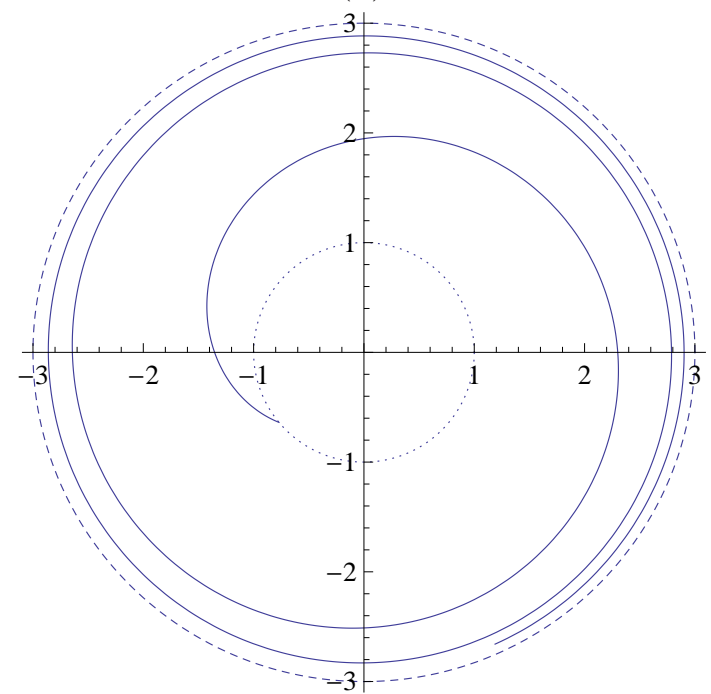

(b)

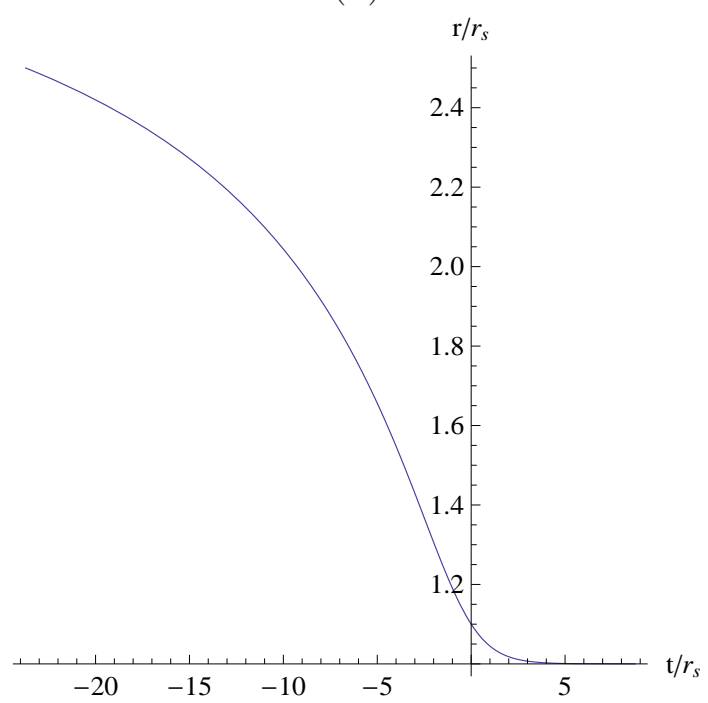

Figure 1: (a) Spatial trajectory of the plunging particle, $r(\phi)$, normalized so that $r_{s}=1$. The dashed line at $r=3$ represents the ISCO, whereas the dotted line at $r=1$ represents the horizon. (b) $r$ coordinate of the plunging particle as a function of coordinate time $t$ (equation (2.10)). 
perturbations reduces to the physics of a $(1+1)$ dimensional wave equation with a nontrivial potential, satisfied by two "master functions" (for odd and even parities) constructed from the metric. These master functions characterize the metric perturbations.

A convenient formalism, suitable for the problem of analyzing gravitational waves generated by material sources, was introduced by Martel and Poisson (2005) [18]. That paper presents gauge-invariant and covariant form of the Regge-Wheeler equation (describing odd-parity perturbations of Schwarzschild space-time) and of the Zerilli equation (describing the even parity perturbations) with two corresponding scalar master functions and invariant source terms constructed from the stress-energy tensor of the matter responsible for the perturbation. In this subsection we review the general theory of Schwarzschild BH perturbations with sources, closely following [18] (see also [19]).

The Schwarzschild metric can be written as

$$
\begin{aligned}
d s^{2}=g_{\mu \nu} d x^{\mu} d x^{\nu} & =g_{a b} d x^{a} d x^{b}+r^{2} \Omega_{A B} d \theta^{A} d \theta^{B} \\
g_{a b} d x^{a} d x^{b} & =-f d t^{2}+f^{-1} d r^{2} \\
\Omega_{A B} d \theta^{A} d \theta^{B} & =d \theta^{2}+\sin ^{2} \theta d \phi^{2}
\end{aligned}
$$

where upper-case latin indices run over the values $2,3(\theta, \phi)$ and lower-case latin indices run over the values $0,1(t, r)$. We study small perturbations around the Schwarzschild space-time, so it is useful to write the metric in the form $g_{\mu \nu}=g_{\mu \nu}+h_{\mu \nu},\left|h_{\mu \nu}\right| \ll 1$. Next, we expand the perturbation in spherical harmonics, according to the transformation laws of the different components under rotation. For the odd-parity sector

$$
\begin{aligned}
h_{a b} & =0 \\
h_{a B} & =\sum_{l m} h_{a}^{l m} X_{B}^{l m} \\
h_{A B} & =\sum_{l m} h_{2}^{l m} X_{A B}^{l m},
\end{aligned}
$$

where $X_{B}^{l m}$ and $X_{A B}^{l m}$ are the odd-parity vector and tensor spherical harmonics ${ }^{7}$, respectively. For the even-parity sector

$$
\begin{aligned}
h_{a b} & =\sum_{l m} h_{a b}^{l m} Y^{l m} \\
h_{a B} & =\sum_{l m} j_{a}^{l m} Y_{B}^{l m} \\
h_{A B} & =r^{2} \sum_{l m}\left(K^{l m} \Omega_{A B} Y^{l m}+G^{l m} Y_{A B}^{l m}\right),
\end{aligned}
$$

where $Y^{l m}, Y_{B}^{l m}$ and $Y_{A B}^{l m}$ are the even-parity scalar, vector and tensor spherical harmonics, respectively. Plugging these expressions into the Einstein equations and using the orthogonality relations of the spherical harmonics, a wave equation for every $(l, m)$ is obtained,

\footnotetext{
${ }^{7}$ More details regarding the spherical harmonics can be found in [18], and in particular we record their normalization conventions: $\int \bar{Y}_{l m} Y_{l^{\prime} m^{\prime}} d \Omega=\delta_{l l^{\prime}} \delta_{m m^{\prime}}, \int \bar{Y}_{l m}^{A} Y_{A}^{l^{\prime} m^{\prime}} d \Omega=l(l+1) \delta_{l l^{\prime}} \delta_{m m^{\prime}}, \int \bar{X}_{l m}^{A} X_{A}^{l^{\prime} m^{\prime}} d \Omega=$ $l(l+1) \delta_{l l^{\prime}} \delta_{m m^{\prime}}, \int \bar{Y}_{l m}^{A B} Y_{A B}^{l^{\prime} m^{\prime}} d \Omega=\frac{1}{2}(l-1) l(l+1)(l+2) \delta_{l l^{\prime}} \delta_{m m^{\prime}}$ and finally $\int \bar{X}_{l m}^{A B} X_{A B}^{l^{\prime} m^{\prime}} d \Omega=\frac{1}{2}(l-1) l(l+$ 1) $(l+2) \delta_{l l^{\prime}} \delta_{m m^{\prime}}$.
} 
satisfied by the corresponding master functions. The Cunningham-Price-Moncrief function, characterizing odd-parity perturbations, is defined by

$$
\psi_{\mathrm{o}}^{l m}:=\frac{2 r}{(l-1)(l+2)} \varepsilon^{a b}\left(\partial_{a} h_{b}^{l m}-\frac{2}{r} r_{a} h_{b}^{l m}\right),
$$

where $\varepsilon^{a b}$ is the Levi-Civita tensor on the $(t, r)$ manifold and $r_{a}=(0,1)$ is the unit co-vector in the $r$ direction. The Cunningham-Price-Moncrief function satisfies the Regge-Wheeler equation

$$
\left(\square-V_{\mathrm{o}}^{l}\right) \psi_{\mathrm{o}}^{l m}=S_{\mathrm{o}}^{l m},
$$

where

$$
V_{\mathrm{o}}^{l}=\frac{l(l+1)}{r^{2}}-\frac{3 r_{s}}{r^{3}}
$$

is the Regge-Wheeler potential, and $S_{\mathrm{o}}^{l m}$ is the source term, on which we will focus later.

The Zerilli-Moncrief function, characterizing even-parity perturbations, is defined by

$$
\psi_{\mathrm{e}}^{l m}:=\frac{2 r}{l(l+1)}\left[\tilde{K}^{l m}+\frac{2}{\Lambda}\left(r^{a} r^{b}\left(h_{a b}^{l m}-2 \nabla_{a}\left(j_{b}-\frac{1}{2} r^{2} \nabla_{b} G\right)\right)-r r^{a} \nabla_{a} \tilde{K}^{l m}\right)\right],
$$

where

$$
\begin{gathered}
\Lambda:=(l-1)(l+2)+\frac{3 r_{s}}{r} \\
\tilde{K}:=K+\frac{1}{2} l(l+1) G-\frac{2}{r} r^{a}\left(j_{a}-\frac{1}{2} r^{2} \nabla_{a} G\right),
\end{gathered}
$$

and $r_{a}=(0,1)$ is the unit co-vector in the $r$ direction on the $(t, r)$ submanifold. The Zerilli-Moncrief function satisfies the Zerilli equation

$$
\left(\square-V_{\mathrm{e}}^{l}\right) \psi_{\mathrm{e}}^{l m}=S_{\mathrm{e}}^{l m},
$$

where

$$
V_{\mathrm{e}}=\frac{1}{\Lambda^{2}}\left[\mu^{2}\left(\frac{\mu+2}{r^{2}}+\frac{3 r_{s}}{r^{3}}\right)+\frac{9 r_{s}^{2}}{r^{4}}\left(\mu+\frac{r_{s}}{r}\right)\right]
$$

is the Zerilli potential $(\mu:=(l-1)(l+2))$ and $S_{\mathrm{e}}^{l m}$ is the source term.

The source terms, as was earlier stated, are constructed from the stress-energy tensor of the material source of gravitational waves. In the odd-parity sector, the source term is given by

$$
S_{\mathrm{o}}^{l m}=-\frac{2 r}{(l-1)(l+2)} \varepsilon^{a b} \nabla_{a} P_{b},
$$

where

$$
P^{a}=\frac{16 \pi r^{2}}{l(l+1)} \int T^{a B} X_{B}^{* l m} d \Omega
$$

and $X^{*}$ denotes the complex conjugate of $X$.

In the even-parity sector, the source term is given by

$$
\begin{aligned}
& S_{\mathrm{e}}=\frac{4}{\Lambda} r_{a} Q^{a}-\frac{1}{r} Q^{\sharp}+\frac{2}{(\mu+2) \Lambda}\left\{-2 r^{2} r^{a} \nabla_{a} Q+\frac{12 r_{s}}{\Lambda} r_{a} r_{b} Q^{a b}+2 r f Q^{b}\right. \\
& \left.+\frac{r}{\Lambda}\left[\mu(\mu-2)+6(\mu-3) \frac{r_{s}}{r}+21 \frac{r_{s}^{2}}{r^{2}}\right] Q\right\},
\end{aligned}
$$


where

$$
\begin{aligned}
Q^{a b} & =8 \pi \int T^{a b} Y^{* l m} d \Omega \\
Q^{a} & =\frac{16 \pi r^{2}}{l(l+1)} \int T^{a B} Y_{B}^{* l m} d \Omega \\
Q^{b} & =8 \pi r^{2} \int T^{A B} \Omega_{A B} Y^{* l m} d \Omega \\
Q^{\sharp} & =\frac{32 \pi r^{4}}{(l-1) l(l+1)(l+2)} \int T^{A B} Y_{A B}^{* l m} d \Omega \\
Q=g^{a b} Q_{a b} . &
\end{aligned}
$$

In this work we ran a check, independent of the derivation of results in [18], of the expressions for the source terms (3.18) and (3.20). The idea is simple - we checked that both sides of the Regge-Wheeler and Zerilli equations with source terms, equations (3.11) and (3.16), agree - when written explicitly as functions of the coefficients of the spherical harmonics in the decomposition of the metric perturbations (e.g. $h_{a}^{l m}, \ldots$ ) appearing in equations (3.4) - (3.9). When substituting expressions (3.10) and (3.13) in the left hand sides of the equations (respectively) such an explicit expression is obtained. For the right hand sides of (3.11), (3.16), we used relations between the coefficients in the spherical harmonic decomposition of the stress-energy tensor ( $P^{a}$ in (3.19), the $Q^{\prime}$ 's in (3.21)-(3.24)) and the metric perturbation coefficients ( $h_{a}^{l m}$, etc..). These relations can be obtained by substituting the decompositions to spherical harmonics in the Einstein equation and equating the different spherical harmonic coefficients. Although the idea is simple, its execution demands some tedious algebra. The process described above was carried out for equations (3.11), (3.16), and the two sides of both equations were found to coincide.

Combining the two wave equations (3.11), (3.16) into a single notation and making the wave operator explicit, we obtain in the frequency domain

$$
\left[\frac{\partial^{2}}{\partial r_{*}^{2}}+\omega^{2}-f V_{\mathrm{o} / \mathrm{e}}^{l}(r)\right] \psi_{\mathrm{o} / \mathrm{e}}^{l m}(r, \omega)=f S_{\mathrm{o} / \mathrm{e}}^{l m}(r, \omega),
$$

where $r_{*}=r+r_{s} \log \left(r / r_{s}-1\right)$ is the usual tortoise coordinate and $\psi_{\mathrm{o} / \mathrm{e}}^{l m}(r, \omega)$ and $S_{\mathrm{o} / \mathrm{e}}^{l m}(r, \omega)$ are the Fourier transforms of the master function and source term, respectively. Notice that changing to derivative with respect to $r_{*}$ instead of $r$ casts the equation explicitly in the form of a flat space $1+1$ dimensional wave equation with a potential. The integration of these equations will be discussed in section 4 .

For later use we record the relation between the master functions and the transversetraceless part of the metric perturbation (which is gauge-invariant) at future null infinity

$$
\begin{aligned}
h_{a b} & =0 \\
h_{a B} & =0 \\
h_{A B} & =r \sum_{l m}\left(\psi_{\mathrm{e}}^{l m} Y_{A B}^{l m}+\psi_{\mathrm{o}}^{l m} X_{A B}^{l m}\right) .
\end{aligned}
$$




\subsection{Plunging mass as a source}

We turn to calculate the source terms corresponding to our plunging point particle. The stress-energy tensor corresponding to a point-like mass is given by

$$
T^{\mu \nu}=\tilde{m} \int d \tau(-g)^{-1 / 2} u^{\mu} u^{\nu} \delta^{(4)}\left(x^{\alpha}-x_{p}^{\alpha}(\tau)\right),
$$

where $\delta^{(4)}\left(x^{\alpha}-y^{\alpha}\right)$ is the four-dimensional Dirac functional, $u^{\mu}$ is the 4 -velocity and $x_{p}^{\alpha}(\tau)$ is the location of the point particle which approximates the compact object. $x_{p}^{\alpha}(\tau)$ are the same functions discussed in section 2 and the added subscript $p$ is to distinguish them in the current context.

In the Schwarzschild space-time, after localizing the $\tau$ integral onto $t_{p}(\tau)$

$$
T^{\mu \nu}=\tilde{m} \frac{f}{\tilde{E}} \frac{u^{\mu}(t) u^{\nu}(t)}{r^{2}} \delta\left(r-r_{p}(t)\right) \delta\left(\theta-\frac{\pi}{2}\right) \delta\left(\phi-\phi_{p}(t)\right) .
$$

To compute the odd-parity source term, we first need to calculate $P^{a}$ (3.19). The integral over the angles is trivial due to the delta functions and we obtain

$$
P^{a}=\frac{16 \pi \tilde{m} \tilde{L} f\left(r_{p}\right)}{l(l+1) \tilde{E} r_{p}^{2}} u^{a}\left(r_{p}\right) \delta\left(r-r_{p}(t)\right) B(l, m) e^{-i m \phi_{p}(t)},
$$

where $B(l, m)$ is defined by

$$
\left.X_{\phi}^{* l m}\right|_{\theta=\frac{\pi}{2}}=B(l, m) e^{-i m \phi}
$$

and can be explicitly written as

$$
B(l, m):=-\sqrt{\frac{2 l+1}{4 \pi} \frac{(l-m) !}{(l+m) !}} \frac{(l+m) ! !}{(l-m-1) ! !} \sin \left(\frac{\pi}{2}(l+m)\right),
$$

for $l>m$ (for $l=m$ replace the factor of $(l-m-1)$ !! in the denominator with $\sqrt{\pi})$.

Equation (3.18) gives us an expression for the odd-parity source term as a function of $r$ and $t$

$$
S_{\mathrm{o}}^{l m}(r, t)=\frac{2 r}{(l-1)(l+2)}\left[f^{-1} \partial_{t} P^{r}+\partial_{r}\left(f P^{t}\right)\right] .
$$

The source term in the frequency domain is given by

$$
S_{\mathrm{o} / \mathrm{e}}^{l m}(r, \omega)=\int_{-\infty}^{\infty} e^{-i \omega t} S_{\mathrm{o} / \mathrm{e}}^{l m}(r, t) d t .
$$

To deal with the time derivative in the integrand of (3.36), coming from the first term in (3.35), we integrate by parts to get a factor of $-i \omega$ from the exponent's time derivative. Now we perform a change of variables in the integral, $t \rightarrow r_{p}(t)$. Additionally, we have explicit analytical expressions for the functions $t_{p}=t_{p}(r)$ (equation (2.10)), appearing in the integrand in the factor $e^{-i \omega t_{p}(r)}$, and $\phi_{p}=\phi_{p}(r)$ (equation (2.7)), appearing in the factor $e^{-i m \phi_{p}(t)}$. Using these relations, all the dependence of the integrand on $r_{p}(t)$ is explicit. Now, using the delta functions, the integral can be evaluated analytically. 
The final result for the frequency domain odd-parity source term is, for $r_{s}<r<3 r_{s}$

$$
\begin{aligned}
S_{\mathrm{o}}^{l m}(r, \omega) & =-\frac{32 \sqrt{3} \pi \tilde{m} r_{s}}{\tilde{\Lambda} r} B(l, m) e^{-i\left(\omega t_{p}(r)+m \phi_{p}(r)\right)} \\
& \times\left[-i \omega\left(9\left(\frac{r}{3 r_{s}-r}\right)^{3}\left(1+3\left(\frac{r_{s}}{r}\right)^{2}\right)\right)-i m \frac{6 \sqrt{6} r_{s} r}{\left(3 r_{s}-r\right)^{3}}+\frac{\sqrt{2} r^{1 / 2}\left(3 r_{s}-4 r\right)}{\left(3 r_{s}-r\right)^{5 / 2}}\right],
\end{aligned}
$$

where $\tilde{\Lambda}=(l-1) l(l+1)(l+2)$. For $r \notin\left(r_{s}, 3 r_{s}\right), S_{\mathrm{o}}^{l m}(r, \omega)=0$.

The calculation of $S_{\mathrm{e}}^{l m}(r, \omega)$ is done in a very similar way (but it is a bit longer). Instead of calculating $P^{a}$ we will need to calculate the $Q$ 's from equations (3.21)-(3.24). We will also write explicit expressions for the relevant spherical harmonics in the $\theta=\frac{\pi}{2}$ plane

$$
\begin{aligned}
& \left.Y^{* l m}\right|_{\theta=\frac{\pi}{2}}=A(l, m) e^{-i m \phi} \\
& \left.Y_{\phi}^{* l m}\right|_{\theta=\frac{\pi}{2}}=-i m A(l, m) e^{-i m \phi} \\
& \left.Y_{\phi \phi}^{* l m}\right|_{\theta=\frac{\pi}{2}}=\left(-m^{2}+\frac{1}{2} l(l+1)\right) A(l, m) e^{-i m \phi},
\end{aligned}
$$

where

$$
A(l, m):=\sqrt{\frac{2 l+1}{4 \pi} \frac{(l-m) !}{(l+m) !}} \frac{(l+m-1) ! !}{(l-m) ! !} \sin \left(\frac{\pi}{2}(l+m+1)\right) .
$$

After calculating the time domain source term by equation (3.20), we Fourier transform it, in the same spirit as in the odd-parity case (using the same change of variables), to obtain an explicit analytical expression.

The final result is, for $r_{s}<r<3 r_{s}$

$$
\begin{aligned}
S_{\mathrm{e}}^{l m}(r, \omega) & =-\frac{16 \pi \tilde{m}}{(\mu+2) \Lambda r^{5 / 2}} A(l, m) e^{-i\left(\omega t_{p}(r)+m \phi_{p}(r)\right)} \\
& \times\left[\frac{9 r r_{s}^{2} \Lambda\left(-2 m^{2}+\mu+2\right)}{\mu\left(3 r_{s}-r\right)^{3 / 2}}+\frac{6 i \sqrt{r}\left(r^{2}+3 r_{s}^{2}\right)\left(3 \sqrt{3} m r_{s}\left(r_{s}-r\right)-2 \sqrt{2} r^{3} w\right)}{\left(3 r_{s}-r\right)^{3}}\right. \\
& -4 i \sqrt{3} m \sqrt{r} r_{s}+\frac{3\left(r^{2}+3 r_{s}^{2}\right)\left(r^{2}(\mu-2) \mu+6 r r_{s}(\mu-3)+21 r_{s}^{2}\right)}{r \Lambda\left(3 r_{s}-r\right)^{3 / 2}} \\
& \left.-\frac{3\left(r-r_{s}\right)\left(4 r^{3}-3 r^{2} r_{s}+24 r r_{s}^{2}-45 r_{s}^{3}\right)}{\left(3 r_{s}-r\right)^{5 / 2}}+\frac{18 r_{s}^{2}\left(r_{s}-r\right)}{\left(3 r_{s}-r\right)^{3 / 2}}-\frac{4 r_{s}\left(3 r_{s}-r\right)^{3 / 2}}{r \Lambda}\right] .
\end{aligned}
$$

For $r \notin\left(r_{s}, 3 r_{s}\right), S_{\mathrm{e}}^{l m}(r, \omega)=0$.

Note that $S_{e}$ is non-zero only for even $l+m$ and similarly for $S_{o}$.

\section{Solving the wave equation}

In this section we discuss the solution to the wave equation (3.26). 


\subsection{Green's function}

Knowing the explicit form of the source terms we proceed to calculate the gravitational waveform. The boundary conditions for equation (3.26) are outgoing waves at infinity

$$
\psi_{\mathrm{o} / \mathrm{e}}^{l m}(r, \omega) \propto e^{-i \omega r_{*}} ; r_{*} \rightarrow \infty,
$$

and ingoing boundary conditions at the horizon

$$
\psi_{\mathrm{o} / \mathrm{e}}^{l m}(r, \omega) \propto e^{i \omega r_{*}} ; r_{*} \rightarrow-\infty
$$

The solution to equation (3.26) is given by

$$
\psi_{\mathrm{o} / \mathrm{e}}^{l m}(r, \omega)=\int G_{\mathrm{o} / \mathrm{e}}^{l}\left(\omega, r_{*}, r_{*}^{\prime}\right) f S_{\mathrm{o} / \mathrm{e}}^{l m}\left(r_{*}^{\prime}, \omega\right) d r_{*}^{\prime},
$$

where $G_{\mathrm{o} / \mathrm{e}}^{l}\left(\omega, r_{*}, r_{*}^{\prime}\right)$ is the frequency domain Green's function given the above-mentioned boundary conditions. It satisfies

$$
\left[\frac{\partial^{2}}{\partial r_{*}^{2}}+\omega^{2}-f V_{\mathrm{o} / \mathrm{e}}^{l}(r)\right] G_{\mathrm{o} / \mathrm{e}}^{l}\left(\omega, r_{*}, r_{*}^{\prime}\right)=\delta\left(r_{*}-r_{*}^{\prime}\right)
$$

with the same boundary conditions.

In order to construct the Green's function we must first define two independent solutions to the homogeneous equation (c.f. [21])

$$
\left[\frac{\partial^{2}}{\partial r_{*}^{2}}+\omega^{2}-f V_{\mathrm{o} / \mathrm{e}}^{l}(r)\right] u_{\mathrm{o} / \mathrm{e}}^{l}\left(\omega, r_{*}\right)=0
$$

Let us denote by $u_{\infty}^{l}$ the solution to (4.5) which satisfies (we will sometimes suppress the parity)

$$
u_{\infty}^{l}\left(r_{*}, \omega\right) \rightarrow e^{-i \omega r_{*}} \quad ; r_{*} \rightarrow+\infty
$$

At the horizon $u_{\infty}$ can be expanded as follows

$$
u_{\infty}^{l}\left(r_{*}, \omega\right) \rightarrow A_{\text {out }}^{l}(\omega) e^{-i \omega r_{*}}+A_{\text {in }}^{l}(\omega) e^{i \omega r_{*}} ; r_{*} \rightarrow-\infty,
$$

where $A_{\text {in }}$ and $A_{\text {out }}$ are some $\omega$ dependent complex coefficients. In a similar manner, we denote by $u_{\text {hor }}^{l}$ the solution to (4.5) which satisfies

$$
u_{\mathrm{hor}}^{l}\left(r_{*}, \omega\right) \rightarrow e^{i \omega r_{*}} ; r_{*} \rightarrow-\infty .
$$

At infinity it can be expanded as

$$
u_{\text {hor }}^{l}\left(r_{*}, \omega\right) \rightarrow B_{\text {out }}^{l}(\omega) e^{-i \omega r_{*}}+B_{\text {in }}^{l}(\omega) e^{i \omega r_{*}} ; r_{*} \rightarrow+\infty
$$

The Green's function satisfies (4.4) with outgoing wave boundary conditions at infinity. Therefore, for $r_{*}>r_{*}^{\prime}$ the solution is proportional to $u_{\infty}^{l}$. Similarly, for $r_{*}<r_{*}^{\prime}$ the solution 
is proportional to $u_{\mathrm{hor}}^{l}$. What remains is to properly match the solutions at $r_{*}=r_{*}^{\prime}$ and obtain the Green's function

$$
G_{\mathrm{o} / \mathrm{e}}^{l}\left(\omega, r_{*}, r_{*}^{\prime}\right)=\left\{\begin{array}{l}
W^{-1}(\omega) u_{\mathrm{hor}}^{l}\left(r_{*}, \omega\right) u_{\infty}^{l}\left(r_{*}^{\prime}, \omega\right) ; r_{*}<r_{*}^{\prime} \\
W^{-1}(\omega) u_{\mathrm{hor}}^{l}\left(r_{*}^{\prime}, \omega\right) u_{\infty}^{l}\left(r_{*}, \omega\right) ; r_{*}>r_{*}^{\prime}
\end{array},\right.
$$

where $W(\omega):=u_{\text {hor }}^{l} \partial_{r_{*}} u_{\infty}^{l}-u_{\infty}^{l} \partial_{r_{*}} u_{\text {hor }}^{l}$ is the Wronskian of $u_{\text {hor }}^{l}, u_{\infty}^{l}$ and is independent of $r_{*}$. Evaluating it both at the horizon and asymptotically we obtain $W(\omega)=-2 i \omega B_{\text {in }}=$ $-2 i \omega A_{\text {out }}$ and in particular $B_{\text {in }}=A_{\text {out }}$.

We are interested in calculating the gravitational waveform measured by a distant observer $\left(r \gg r_{s}\right)$. We can, therefore, simplify the Green's function (4.10)

$$
G_{\mathrm{o} / \mathrm{e}}^{l}\left(\omega, r_{*}, r_{*}^{\prime}\right) \simeq-\frac{1}{2 i \omega B_{i n}} e^{-i \omega r_{*}} u_{\mathrm{hor}}^{l}\left(r_{*}^{\prime}, \omega\right)
$$

Knowing the Green's function (4.10), (4.11) we can proceed to express the solution to the wave equation. Using (4.3) and transforming back to the time domain we have

$$
\psi_{\mathrm{o} / \mathrm{e}}^{l m}(r, t)=\frac{1}{2 \pi} \int_{r_{s}}^{3 r_{s}} d r^{\prime}\left[\int_{-\infty}^{\infty} e^{i \omega t} G_{\mathrm{o} / \mathrm{e}}^{l}\left(\omega, r_{*}, r_{*}^{\prime}\right) S_{\mathrm{o} / \mathrm{e}}^{l m}\left(r_{*}^{\prime}, \omega\right) d \omega\right] .
$$

\subsection{Ringdown amplitudes}

It is known that as a perturbed black hole settles back to its stationary state the background metric exhibits an exponentially decaying radiation known as ringdown, or quasi-normal modes. This can be seen from the analytic structure of Green's functions for the ReggeWheeler and Zerilli equations in the complex frequency domain. It is known (e.g.[22]) that these Green's functions have poles in the upper half plane $(\operatorname{Im}(\omega)>0)$ and a branch cut along the positive part of the $\operatorname{Im}(\omega)$ axis as shown schematically in figure 2 . The poles are interpreted as the quasi-normal modes. Their locations are the quasi-normal frequencies, whose imaginary part is responsible for their decaying nature at late times. The branch cut is interpreted as the "tail" resulting from scattering the waves off the background geometry at large radii. Here we shall proceed to derive a formula for the ringdown or quasi-normal mode amplitudes. Concentrating on them is justified since they are known to dominate over the tail for late times but not too late times - time region (iv) of [22], the discussion after eq. (46). See [23, 24, 25, 26] for further discussion. In addition, these amplitudes are one of the ingredients which go into current waveform models, as reviewed in the introduction, but as of now they are matched (with a different part of the modeled waveform) rather than computed from the theory.

We now turn to evaluate the integral in square brackets in (4.12), utilizing the continuation to complex frequencies. We deform the contour from the real axis to parts (b) and (c) in figure 2 and collect the residue contribution from the poles.

The poles correspond to zeros of the Wronskian. We denote by $\omega_{n l}$ the $n$-th pole for a given $l$ (ordered by increasing imaginary part). At these frequencies $B_{\text {in }}\left(\omega_{n l}\right)=A_{\text {out }}\left(\omega_{n l}\right)=$ 0 and the leading behavior for $B_{\text {in }}$ is

$$
\left.B_{\mathrm{in}} \simeq \partial_{\omega} B_{\mathrm{in}}\right|_{\omega=\omega_{n l}}\left(\omega-\omega_{n l}\right) .
$$




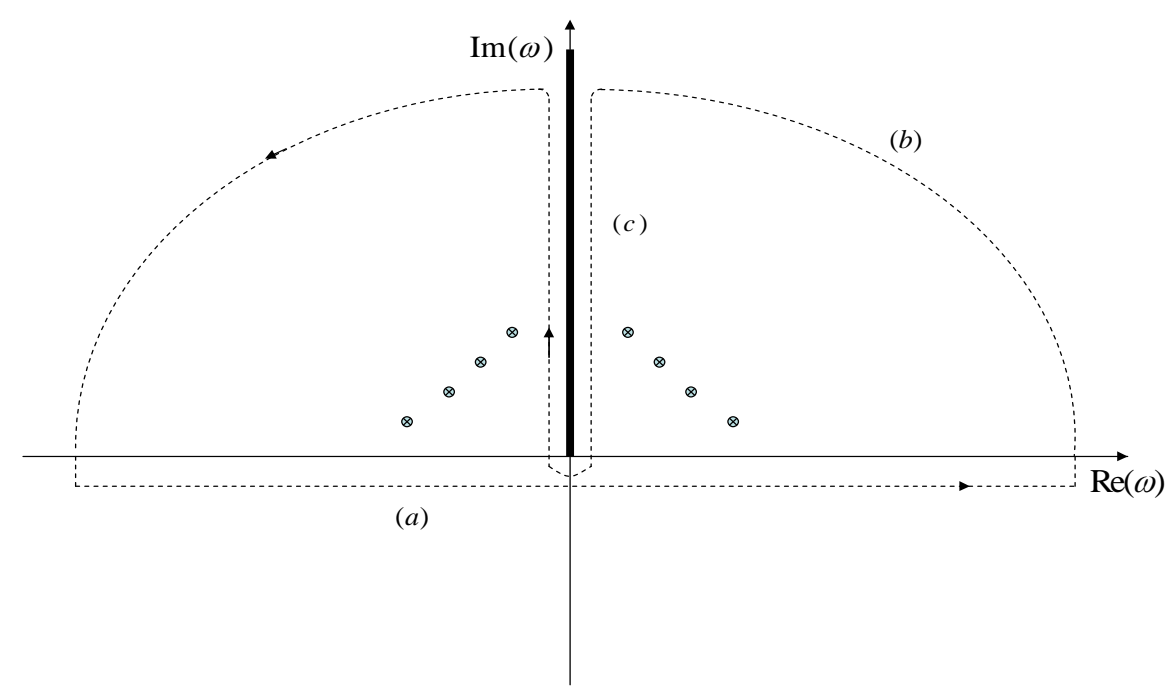

Figure 2: Analytic structure of the Green's function in the upper half $\omega$ plane and the integration contour. The thick line represents the branch cut on the positive $\operatorname{Im}(\omega)$ axis. The dashed line represents the integration contour. The dots represent some of the Green's function's poles at the QNM frequencies.

For convenience we assign notation to these constants

$$
\beta_{n l}:=-\left.\partial_{\omega} B_{\text {in }}\right|_{\omega=\omega_{n l}} .
$$

Their formalism and numerical computation can be found at [27].

The $r_{*} \gg r_{s}$ Green's function (4.11) (implying $r_{*}>r_{*}^{\prime}$ ) for $\omega \approx \omega_{n l}$ can now be written as

$$
G_{\mathrm{o} / \mathrm{e}}^{l}\left(\omega, r_{*}, r_{*}^{\prime}\right)=\frac{e^{-i \omega_{n l} r_{*}} u_{\mathrm{hor}}^{l}\left(r_{*}^{\prime}\right)}{2 i \omega_{n l} \beta_{n l}\left(\omega-\omega_{n l}\right)} .
$$

At late times when the contribution of parts (b) vanishes and part (c) is neglected as we discussed above the integral over frequencies (4.12) can be approximated by the sum over the residues

$$
\psi_{\mathrm{o} / \mathrm{e}}^{l m}(r, t)=\left.\frac{1}{2 \pi} \int_{r_{s}}^{3 r_{s}} d r^{\prime} \sum_{n} 2 \pi i \operatorname{Res}\right|_{\omega=\omega_{n l}}\left[e^{i \omega t} G_{\mathrm{o} / \mathrm{e}}^{l}\left(\omega, r_{*}, r_{*}^{\prime}\right) S_{\mathrm{o} / \mathrm{e}}^{l m}\left(r_{*}^{\prime}, \omega\right)\right] .
$$

Plugging in (4.15), we obtain

$$
\psi_{\mathrm{o} / \mathrm{e}}^{l m}(r, t)=\sum_{n} \frac{e^{i \omega_{n l}\left(t-r_{*}\right)}}{2 \omega_{n l} \beta_{n l}} \int_{r_{s}}^{3 r_{s}} u_{\mathrm{hor}}^{l}\left(r_{*}^{\prime}\right) S_{\mathrm{o} / \mathrm{e}}^{l m}\left(r_{*}^{\prime}, \omega_{n l}\right) d r^{\prime},
$$


where the index $n$ runs over all QNM's for a given $l$.

We identify the coefficient of $e^{i \omega_{n l}\left(t-r_{*}\right)}$ with the ringdown amplitude

$$
R_{n l m}:=\frac{1}{2 \omega_{n l} \beta_{n l}} \int_{r_{s}}^{3 r_{s}} u_{\mathrm{hor}}^{l}\left(r_{*}^{\prime}\right) S_{\mathrm{o} / \mathrm{e}}^{l m}\left(r_{*}^{\prime}, \omega_{n l}\right) d r^{\prime} .
$$

This is our main result for the ringdown amplitudes.

For $r \rightarrow r_{\text {ISCO }} u_{\text {hor }}$ is finite and the third order poles in the source $S$ are suppressed by the term $\exp (-i \omega t)$ which is bounded by $|\exp (-i \omega t)| \leq \exp (\operatorname{Im}(\omega) t) \simeq \exp [\operatorname{Im}(\omega)$. const $/ \sqrt{r-r_{\text {ISCO }}}$ and so the integrand vanishes exponentially in the limit.

In the limit $r \rightarrow r_{\text {hor }}$, however, the wavefunction diverges

$$
u_{\text {hor }} \propto \exp \left(i \omega r_{*}\right) \propto\left(\frac{r}{r_{s}}-1\right)^{i \frac{\omega}{r_{s}}} .
$$

The source term, too, diverges in this limit in the same fashion. At $r \rightarrow r_{s}$ the trajectory satisfies $r_{*} \simeq t+$ const and so

$$
S_{o / e} \propto \exp (-i \omega t) \propto \exp \left(i \omega r_{*}\right) \propto\left(\frac{r}{r_{s}}-1\right)^{i \frac{\omega}{r_{s}}} .
$$

Altogether, then, the integrand is $\propto\left(\frac{r}{r_{s}}-1\right)^{2 i \frac{\omega}{r_{s}}}$ and so the integral diverges when $\operatorname{Im}\left(\frac{\omega}{r_{s}}\right) \geq$ $\frac{1}{2}$. This spurious divergence needs to be regularized. This problem of a diverging QNM excitation integral was realized already in [22] for different excitation scenarios. Two different methods (equivalent in principle) to regularize the integral are discussed there, one of them being the divergence subtraction method (introduced in [29] for excitation integrals of non-QNM frequencies). This is the method we used in practice when evaluating the amplitudes numerically, and it will be discussed in the next section.

\section{Numerical Evaluation}

In this section we numerically evaluate the amplitudes (4.18).

\subsection{Method}

We started by calculating the numerical values of the QNM frequencies $\omega_{n l}$. One can use either one of several methods (see the review [28]), and we chose Leaver's continued fraction method (see [22]) which can be extended to yield also the QNM wavefunctions $u_{\text {hor }}\left(\omega_{n l}, r\right)$. More specifically, the wavefunctions are obtained in the regime of interest as a powerseries expansion in $\left(1-\frac{r_{s}}{r}\right)$ multiplying a factored-out non-analytical part. The calculated frequencies were confirmed by the literature, and the wavefunctions were confirmed by substitution into the homogeneous Regge-Wheeler and Zerilli equations. We continued to calculate the excitation factors (or transmission residues) $\beta_{n l}$, again using Leaver's method, and the first few n's and l's were confirmed by Leaver's results. We found [27] to be a very useful and detailed guide to the calculation of the excitation factors (there the more general rotating $\mathrm{BH}$ case is considered). 
The next ingredient in the integrand is the source term $S_{\mathrm{o} / \mathrm{e}}^{l m}\left(r_{*}, \omega_{n l}\right)$, which is given analytically by (3.38), (3.43) where one should substitute the trajectory functions $t_{p}(r)$ (2.10) and $\phi_{p}(r)(2.7)$.

It remains to perform the numerical integration in (4.18). As noted in the end of last section, this integral diverges and must be regularized by what is essentially an analytic continuation. To do so, we used the so-called divergence subtraction method ([29],[22]). The idea is the following: we set the integrand to zero at the horizon $\left(r \rightarrow r_{\text {hor }}\right)$ by subtracting from it a function $f$ that behaves the same as the integrand as $r \rightarrow r_{\text {hor }}$, thereby getting rid of the unphysical divergence at that integration limit. In order not to add anything finite to the integral we will need to add the value of $F:=\int f d r$ at the upper limit of integration. The integration constant in $F$ is determined by the condition that $F$ does not have a part $\sim$ const. as $r \rightarrow 1$. That is, if $f$ is of the form given below in (5.2), we integrate it using the rule $\int r^{\alpha} d r=\frac{r^{\alpha+1}}{\alpha+1}$ without the addition of a constant. In summary

$$
\left.\int_{r_{s}}^{3 r_{s}} u_{\mathrm{hor}}^{l}\left(r_{*}^{\prime}\right) S_{\mathrm{o} / \mathrm{e}}^{l m}\left(r_{*}^{\prime}, \omega_{n l}\right) d r^{\prime}\right|_{\text {physical part }}=\int_{r_{s}}^{3 r_{s}}\left[u_{\mathrm{hor}}^{l}\left(r_{*}^{\prime}\right) S_{\mathrm{o} / \mathrm{e}}^{l m}\left(r_{*}^{\prime}, \omega_{n l}\right)-f\right] d r^{\prime}+\left.F\right|_{3 r_{s}} .
$$

$f$ is, of course, not unique. Any function which will be easy to integrate analytically (and behaves in the desirable way as $r \rightarrow r_{\text {hor }}$ ) will do. The (natural) $f$ we chose to use was

$$
f=\left(\frac{r}{r_{s}}-1\right)^{2 i \frac{\omega}{r_{s}}}\left(A_{0}+\left(A_{1}\left(r-r_{s}\right)+\ldots\right)\right.
$$

where the coefficients $A_{i}$ are determined from our expressions for $u_{\mathrm{hor}}^{l}$ and $S_{\mathrm{o} / \mathrm{e}}^{l m}$ and enough terms must be taken as to assure convergence.

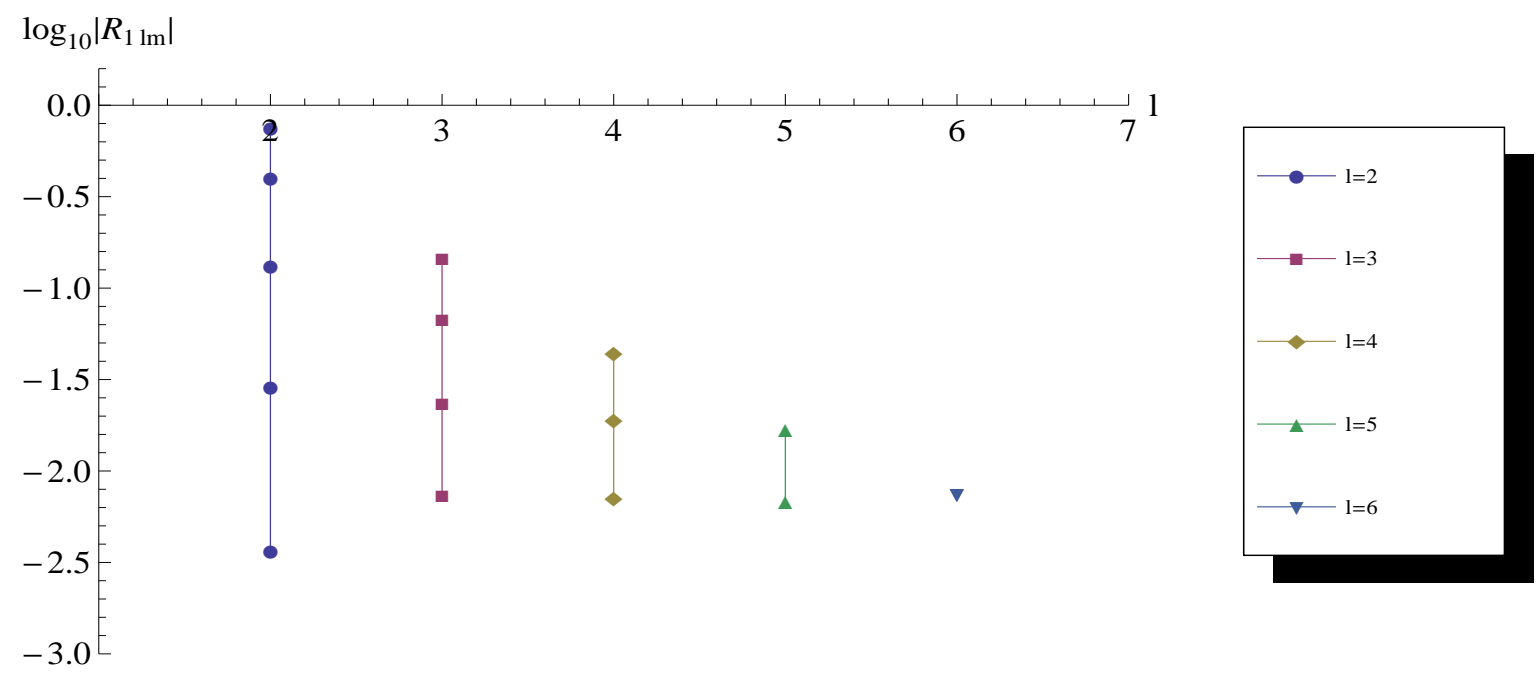

Figure 3: Ringdown amplitudes from tables 1,2 depicted on a logarithmic scale for leading $n=1$, $2 \leq l \leq 6$ and several $m$ values. The amplitudes decrease as $l-m$ increases, for instance for $l=3$ the top point corresponds to $m=3$ while the bottom one to $m=0$. 


\subsection{Results}

From equation (4.17) it is clear that the ringdown amplitudes are defined only up to a shift in the time coordinate; if we take $t \rightarrow t+t^{\prime}$ the amplitudes will transform as

$$
R_{n l m} \rightarrow R_{n l m} e^{i \omega_{n l} t^{\prime}}
$$

We fix $t_{0}$, the integration constant in equation (2.10), somewhat arbitrarily such that $r(t=0)=1.1 r_{s}$, which we observed to be shortly after the onset of ringdown [30], namely the region which is well described by (4.16).

It is worthwhile to note that the decay constants (imaginary parts of the frequencies $\left.\omega_{n l}\right)$ do not vary with $m$ and vary only little with $l$ while they vary strongly with overtone number $n$ and hence (5.3) shifting $t_{0}$ will not affect the ratio of magnitudes of amplitudes which differ only by their $m$, it will weakly affect different $l$ 's and finally strongly affect the ratio for different $n$ 's. Note also that the real signal is gotten by adding the complex conjugate to sum of ringdown exponentials. This is equivalent to the doubling of QNM frequencies $\Re(\omega) \rightarrow-\Re(\omega)$ as in figure 2 .

Results for amplitudes of the first few dominant modes are displayed in table (1). In table (2) the contribution of higher $l$ 's is displayed, up to $l=10$. In table (3) numerical values of QNM frequencies are displayed for the first few $(n, l)$. Figure 3 gives a graphic illustration of several amplitudes on a logarithmic scale.

In figure 4 we display the ringdown waveform (calculated from the amplitudes through equations (4.16), (3.29)), as viewed by observers in different angular positions. Notice
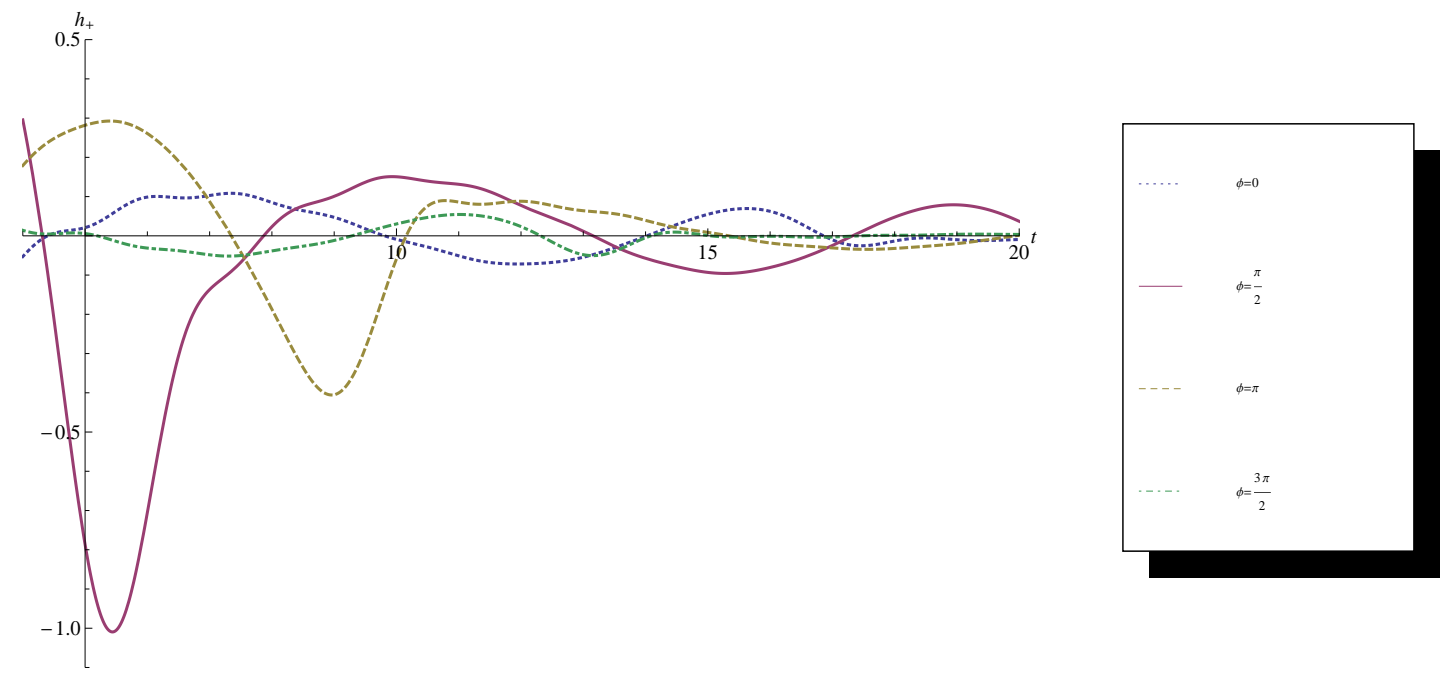

Figure 4: Ringdown waveforms summing the dominant $(l, m, n)$ contributions. The (distant) observer is situated in the equatorial plane $\left(\theta=\frac{\pi}{2}\right)$, at: (a) $\phi=0$, (b) $\phi=\frac{\pi}{2}$, (c) $\phi=\pi$, (d) $\phi=\frac{3 \pi}{2}$. The horizontal axis represents $t-r$ in units of $r_{s}$, while the vertical axis represents $h_{+}$, the + polarized part of the gravitational wave normalized by $r / \tilde{m}$. Notice the asymmetric nature of the ringdown waveform. 
the highly asymmetric nature of the ringdown waveform - observers in different azimuthal positions see very different waveforms. This is due to the fact that the trajectory breaks azimuthal symmetry, by infalling at some specific angular position. Of course, waveforms for observers in any angular position can be easily calculated from the amplitudes, via equation (3.29).

\section{Acknowledgements}

We thank Amos Ori, Umpei Miyamoto, Omer Bromberg and Gerhard Schäfer for comments on the manuscript. We thank Emanuele Berti and Vitor Cardoso for collaboration on a related paper [30].

This research is supported by The Israel Science Foundation grant no 607/05, by the German Israel Cooperation Project grant DIP H.52, and the Einstein Center at the Hebrew University.

\section{References}

[1] S. Rowan and J. Hough, "Gravitational wave detection by interferometry (ground and space)," Living Rev. Rel. 3, 3 (2000). B. S. Sathyaprakash and B. F. Schutz, "Physics, astrophysics and cosmology with gravitational waves," Living Rev. Rel. 12, 2 (2009) [arXiv:0903.0338 [gr-qc]].

[2] F. Pretorius, "Binary black hole coalescence," arXiv:0710.1338 [gr-qc].

[3] A. Ori and K. S. Thorne, "The transition from inspiral to plunge for a compact body in a circular equatorial orbit around a massive, spinning black hole," Phys. Rev. D 62, 124022 (2000) [arXiv:gr-qc/0003032].

[4] L. Blanchet, "Gravitational radiation from post-Newtonian sources and inspiralling compact binaries," Living Rev. Rel. 5, 3 (2002), update: Living Rev. Rel. 9, 4 (2006) [arXiv:gr-qc/0202016]. "Post-Newtonian theory and the two-body problem," arXiv:0907.3596 [gr-qc].

[5] A. Buonanno and T. Damour, "Effective one-body approach to general relativistic two-body dynamics," Phys. Rev. D 59, 084006 (1999) [arXiv:gr-qc/9811091].

[6] A. Buonanno and T. Damour, "Transition from inspiral to plunge in binary black hole coalescences," Phys. Rev. D 62, 064015 (2000) [arXiv:gr-qc/0001013].

[7] T. Damour and A. Nagar, "The Effective One Body description of the Two-Body problem," arXiv:0906.1769 [gr-qc].

[8] A. Nagar, T. Damour and A. Tartaglia, "Binary black hole merger in the extreme mass ratio limit," Class. Quant. Grav. 24, S109 (2007) [arXiv:gr-qc/0612096].

[9] T. Damour and A. Nagar, "Faithful Effective-One-Body waveforms of small-mass-ratio coalescing black-hole binaries," Phys. Rev. D 76, 064028 (2007) [arXiv:0705.2519 [gr-qc]].

[10] Y. Mino and J. Brink, "Gravitational radiation from plunging orbits - perturbative study -," Phys. Rev. D 78, 124015 (2008) [arXiv:0809.2814 [gr-qc]].

[11] P. C. Peters, "Gravitational radiation and the motion of two point masses," Phys. Rev. 136, B1224 (1964). 
[12] L. Barack and C. Cutler, "LISA capture sources: Approximate waveforms, signal-to-noise ratios, and parameter estimation accuracy," Phys. Rev. D 69, 082005 (2004) [arXiv:gr-qc/0310125].

[13] C. Hopman and T. Alexander, "The orbital statistics of stellar inspiral and relaxation near a massive black hole: Characterizing gravitational wave sources," Astrophys. J. 629, 362 (2005) [arXiv:astro-ph/0503672].

[14] P. Amaro-Seoane, J. R. Gair, M. Freitag, M. Coleman Miller, I. Mandel, C. J. Cutler and S. Babak, "Astrophysics, detection and science applications of intermediate- and extreme mass-ratio inspirals," Class. Quant. Grav. 24, R113 (2007) [arXiv:astro-ph/0703495].

[15] S. Chandrasekhar, "The mathematical theory of black holes," Oxford, UK: Clarendon (1992) $646 \mathrm{p}$.

[16] T. Regge and J. A. Wheeler, "Stability of a schwarzschild singularity," Phys. Rev. 108 (1957) 1063.

[17] F. J. Zerilli, "Effective potential for even parity Regge-Wheeler gravitational perturbation equations," Phys. Rev. Lett. 24 (1970) 737.

[18] K. Martel and E. Poisson, "Gravitational perturbations of the Schwarzschild spacetime: A practical covariant and gauge-invariant formalism," Phys. Rev. D 71 (2005) 104003 [arXiv:gr-qc/0502028].

[19] Y. Mino, M. Sasaki, M. Shibata, H. Tagoshi and T. Tanaka, "Black hole perturbation," Prog. Theor. Phys. Suppl. 128, 1 (1997) [arXiv:gr-qc/9712057].

[20] T. Apostolatos, D. Kennefick, E. Poisson and A. Ori, "Gravitational radiation from a particle in circular orbit around a black hole. 3: Stability of circular orbits under radiation reaction," Phys. Rev. D 47, 5376 (1993).

[21] G. Arfken, "Mathematical methods for physicists ," Academic (1985).

[22] E. W. Leaver, "Spectral decomposition of the perturbation response of the Schwarzschild geometry," Phys. Rev. D 34 (1986) 384.

[23] N. Andersson, "Evolving test-fields in a black-hole geometry," Phys. Rev. D 55 (1997) 468 [arXiv:gr-qc/9607064].

[24] E. S. C. Ching, P. T. Leung, W. M. Suen and K. Young, "Wave propagation in gravitational systems: Late time behavior," Phys. Rev. D 52 (1995) 2118 [arXiv:gr-qc/9507035].

[25] S. Hod and T. Piran, "Late-time evolution of charged gravitational collapse and decay of charged scalar hair. I," Phys. Rev. D 58, 024017 (1998) [arXiv:gr-qc/9712041].

[26] L. Barack and A. Ori, "Late-time decay of scalar perturbations outside rotating black holes," Phys. Rev. Lett. 82, 4388 (1999) [arXiv:gr-qc/9902082].

[27] E. Berti and V. Cardoso, "Quasinormal ringing of Kerr black holes. I: The excitation factors," Phys. Rev. D bf 74, 104020 (2006) [arXiv:gr-qc/0605118].

[28] E. Berti, V. Cardoso and A. O. Starinets, "Quasinormal modes of black holes and black branes," Class. Quant. Grav. 26, 163001 (2009) [arXiv:0905.2975 [gr-qc]].

[29] S. L. Detweiler and E. Szedenits, "Black Holes And Gravitational Waves. Ii. Trajectories Plunging Into A Nonrotating Hole," Astrophys. J. 231, 211 (1979).

[30] E. Berti, V. Cardoso, S. Hadar and B. Kol, in progress. 
Table 1: Numerical values of the ringdown amplitudes $R_{n l m}$ for $l=2,3,4,5$, several values of the overtone number $n$ and all values of $m$. Conventions: $t_{0}$ (2.10) was taken such that the plunging object is at $1.1 r_{s}$ at $t=0$, the overall normalization is defined by $(3.10,3.13)$, and the normalization of spherical harmonics is given in footnote 7 .

\begin{tabular}{cccc}
\multicolumn{4}{c}{$l=2$} \\
\hline$m$ & $n=1$ & $n=2$ & $n=3$ \\
\hline 2 & $-0.0985724-0.747787 i$ & $-0.229354+0.428849 i$ & $0.167484-0.251937 i$ \\
1 & $-0.0210521+0.399297 i$ & $0.441304-0.31877 i$ & $-0.489451+0.053276 i$ \\
0 & $-0.0887841+0.0979244 i$ & $0.303357+0.0416042 i$ & $-0.249012-0.314569 i$ \\
-1 & $0.0274099+0.00889306 i$ & $-0.0324417-0.0903849 i$ & $-0.0867015+0.154488 i$ \\
-2 & $(-0.735088+3.59504 i) \times 10^{-3}$ & $0.0135078-0.0089118 i$ & $-0.0394189-0.010878 i$ \\
\hline \hline
\end{tabular}

\begin{tabular}{cccc}
\multicolumn{4}{c}{$l=3$} \\
\hline$m$ & $n=1$ & $n=2$ & $n=3$ \\
\hline 3 & $-0.0375476+0.141024 i$ & $0.117107-0.057957 i$ & $-0.0862334+0.00839625 i$ \\
2 & $0.0336116-0.0585892 i$ & $-0.104903+0.00738472 i$ & $0.0855561+0.0540601 i$ \\
1 & $0.0186212-0.0145793 i$ & $-0.0499562-0.0209467 i$ & $0.0245107+0.0656791 i$ \\
0 & $(-7.36412+0.205584 i) \times 10^{-3}$ & $0.0119392+0.0185584 i$ & $0.0108096-0.0349259 i$ \\
-1 & $(-1.32958-1.74202 i) \times 10^{-3}$ & $-2.4849+7.94279 i) \times 10^{-3}$ & $0.0160937-0.00679286 i$ \\
-2 & $(-3.59688+3.99339 i) \times 10^{-4}$ & $(2.43071+0.097078 i) \times 10^{-3}$ & $(-3.98823-4.75782 i) \times 10^{-3}$ \\
-3 & $(-5.2293-6.09408 i) \times 10^{-5}$ & $(-0.284844+4.19003 i) \times 10^{-4}$ & $(9.4908-8.54244 i) \times 10^{-4}$ \\
\hline \hline
\end{tabular}

\begin{tabular}{ccc} 
& & $l=4$ \\
\hline$m$ & $n=1$ & $n=2$ \\
\hline 4 & $0.0318095-0.0308272 i$ & $-0.0489266-0.00923324 i$ \\
3 & $-0.0165716+0.00919402 i$ & $0.0289206+0.0167393 i$ \\
2 & $(-6.86211+1.83095 i) \times 10^{-3}$ & $0.0114492+0.0128208 i$ \\
1 & $(2.32275+0.593617 i) \times 10^{-3}$ & $(-1.76906-7.17998 i) \times 10^{-3}$ \\
0 & $(4.47817+5.89624 i) \times 10^{-4}$ & $(1.10773-2.61122 i) \times 10^{-3}$ \\
-1 & $(0.514994-2.12774 i) \times 10^{-4}$ & $(-9.22639+3.19073 i) \times 10^{-4}$ \\
-2 & $(6.24213-1.42382 i) \times 10^{-5}$ & $(-2.49301-2.12194 i) \times 10^{-4}$ \\
-3 & $(-0.482705-1.61408 i) \times 10^{-5}$ & $(-5.20619+8.05728 i) \times 10^{-5}$ \\
-4 & $(2.7626-0.0853029 i) \times 10^{-6}$ & $(-1.26026-1.17755 i) \times 10^{-5}$ \\
\hline \hline
\end{tabular}

$$
l=5
$$

\begin{tabular}{cc}
\hline$m$ & $n=1$ \\
\hline 5 & $-0.0166349+0.00302628 i$ \\
4 & $(6.80123+0.509818 i) \times 10^{-3}$ \\
3 & $(2.61522+0.629434 i) \times 10^{-3}$ \\
2 & $(-7.32533-5.82304 i) \times 10^{-4}$ \\
1 & $(-1.14018-2.79764 i) \times 10^{-4}$ \\
0 & $(-3.06403+8.96566 i) \times 10^{-5}$ \\
\hline \hline
\end{tabular}


Table 2: Numerical values of the ringdown amplitudes $R_{n l m}$ for $l=6$ to 10 , overtone number $n=1$ and dominant $m$ 's.

\begin{tabular}{ccc}
\hline$l$ & $m=l$ & $m=l-1$ \\
\hline 6 & $(6.73817+3.1156 i) \times 10^{-3}$ & $(-2.21976-1.78938 i) \times 10^{-3}$ \\
7 & $(-1.75245-3.12505 i) \times 10^{-3}$ & $(0.356901+1.26881 i) \times 10^{-3}$ \\
8 & $(-0.229465+1.83699 i) \times 10^{-3}$ & $(2.27002-6.14468 i) \times 10^{-4}$ \\
9 & $(6.97897-7.26025 i) \times 10^{-4}$ & $(-2.87291+1.88928 i) \times 10^{-4}$ \\
10 & $(-4.03242-3.58438 i) \times 10^{-4}$ & $(1.04411+1.44422 i) \times 10^{-4}$ \\
& & \\
\hline \hline
\end{tabular}

Table 3: Numerical values of some dominant QNM frequencies in units of $r_{s}^{-1}$.

\begin{tabular}{cccc}
\hline$l$ & $n=1$ & $n=2$ & $n=3$ \\
\hline 2 & $-0.74734+0.17792 i$ & $-0.69342+0.54783 i$ & $-0.60210+0.95655 i$ \\
3 & $-1.19889+0.18540 i$ & $-1.16529+0.56259 i$ & $-1.10337+0.95818 i$ \\
4 & $-1.61836+0.18832 i$ & $-1.59326+0.56866 i$ & $-1.54542+0.95981 i$ \\
5 & $-2.02459+0.18974 i$ & & \\
6 & $-2.42402+0.19053 i$ & & \\
& & & \\
\hline \hline
\end{tabular}

